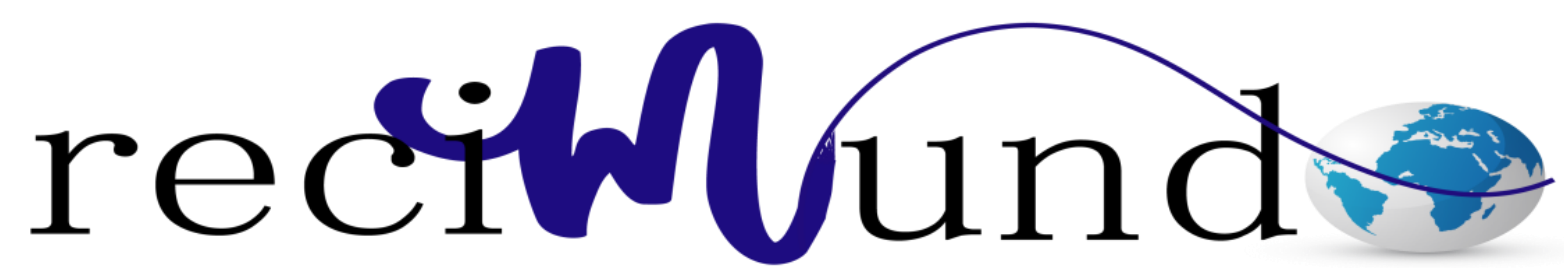

Revista Científica Mundo de la Investigación y el Conocimiento

\author{
Karla Valeria Arcentales Vera a; Melissa Mercedes Mendieta Torres ${ }^{\text {b; }}$ César \\ Enrique Vera Sauhing ${ }^{c}$; Diego Israel Ojeda Maldonado ${ }^{d}$
}

Diagnóstico de las patologías de tubo digestivo y anexos para proceder al manejo clínico y quirúrgico en el tratamiento adecuado

Diagnosis of the pathologies of the digestive tract and annexes to proceed to the clinical and surgical management in the appropriate treatment

Revista Científica Mundo de la Investigación y el Conocimiento. Vol. 3 núm. 4., diciembre, ISSN: 2588-073X, 2019, pp. 363-398

DOI: $10.26820 /$ recimundo/3.(4).diciembre.2019.363-398

URL: http://recimundo.com/index.php/es/article/view/678

Código UNESCO: 3205 Medicina Interna

Tipo de Investigación: Artículo de Revisión

(C) RECIMUNDO; Editorial Saberes del Conocimiento, 2019

Recibido: 15/09/2019

Aceptado: 23/11/2019

Publicado: 30/12/2019

Correspondencia: karinaborjaa@gmail.com

a. Médico; Investigadora Independiente; Guayaquil, Ecuador; karlavaleria-10@live.com

b. Médico; Investigadora Independiente; Guayaquil, Ecuador; melissamendieta@ hotmail.com

c. Médico; Investigador Independiente; Guayaquil, Ecuador; csauhing@gmail.com

d. Médico; Investigador Independiente; Loja, Ecuador; dieguisrael1994@gmail.com 


\section{Diagnóstico de las patologías de tubo digestivo y anexos para proceder al manejo clínico y quirúrgico en el tratamiento adecuado}

Vol. 3, núm. 4., (2019)

Karla Valeria Arcentales Vera; Melissa Mercedes Mendieta Torres; César Enrique Vera Sauhing; Diego Israel Ojeda Maldonado

\section{RESUMEN}

El Aparato digestivo comprende todo lo relacionado con la admisión de alimentos, es decir, los órganos que intervienen en el viaje que realiza el alimento: labios, encías, dientes, lengua; con su transporte: faringe y esófago; con su digestión: estómago, intestino, páncreas, hígado: y por último, con su expulsión hacia el exterior: región anal. Se trata de órganos muy importantes que originan con facilidad desequilibrios funcionales que no hay que pasar por alto. Cuando una patología afecta a uno de estos aparatos, a menudo se ve envuelta también la nutrición, produciéndose un decaimiento general y, en los casos graves, la muerte. En esencia, la función principal del tubo digestivo consiste en proporcionar al organismo vivo el adecuado suministro de material (agua, electrolitos, nutrientes) que necesita para asegurar su propio mantenimiento como tal, de forma que ello le permita obtener la energía y materia que, por su carácter dinámico, requiere para llevar a cabo los procesos vitales, así como los de reposición estructural, crecimiento y reproducción. Para cumplir con este cometido, en el aparato digestivo se producen, de manera esquemática, los siguientes hechos fisiológicos fundamentales: a) Movimiento del material a través del tracto gastrointestinal, desde un extremo a otro y en sentido distal. La función motora que lo regula es de una importancia capital para el conjunto del sistema, ya que supone en la práctica, el factor básico que condiciona la correcta operatividad de los otros. b) Secreción de los jugos digestivos. c) Digestión enzimática. d) Absorción o paso a través de la barrera intestinal, de las substancias digeridas, agua y electrolitos. El funcionalismo correcto del que se hizo referencia exige la integridad anatómica y estructural de este tubo digestivo. Las principales capas en las que genéricamente se constituye, del interior al exterior, es decir, hacia la luz intestinal, dejando al margen características particulares de cada una de las partes que lo componen.

Palabras Claves: Patologías de Tubo Digestivo; Manejo Clínico; Tratamiento; Manejo Quirúrgico; Tracto Gastrointestinal. 


\title{
Diagnóstico de las patologías de tubo digestivo y anexos para proceder al manejo clínico y quirúrgico en el tratamiento adecuado
}

Vol. 3, núm. 4., (2019)

Karla Valeria Arcentales Vera; Melissa Mercedes Mendieta Torres; César Enrique Vera

Sauhing; Diego Israel Ojeda Maldonado

\begin{abstract}
The digestive system includes everything related to the admission of food, that is, the organs involved in the journey that makes the food: lips, gums, teeth, tongue; with its transport: pharynx and esophagus; with its digestion: stomach, intestine, pancreas, liver: and finally, with its expulsion outward: anal region. These are very important organs that easily cause functional imbalances that should not be overlooked. When a pathology affects one of these devices, nutrition is often also involved, causing a general decay and, in severe cases, death. In essence, the main function of the digestive tract is to provide the living organism with the adequate supply of material (water, electrolytes, nutrients) that it needs to ensure its own maintenance as such, so that it allows it to obtain the energy and matter that, Due to its dynamic nature, it requires the carrying out of vital processes, as well as those of structural replacement, growth and reproduction. To fulfill this task, the following fundamental physiological facts occur schematically in the digestive system: a) Movement of the material through the gastrointestinal tract, from one end to the other and distally. The motor function that regulates it is of paramount importance for the whole system, since it supposes in practice, the basic factor that conditions the correct operability of others. b) Secretion of digestive juices. c) Enzymatic digestion. d) Absorption or passage through the intestinal barrier of digested substances, water and electrolytes. The correct functionalism referred to requires the anatomical and structural integrity of this digestive tract. The main layers in which it is generically constituted, from the inside to the outside, that is, towards the intestinal lumen, leaving aside particular characteristics of each of the parts that compose it.
\end{abstract}

Palabras Claves: Digestive Tube Pathologies; Clinical Management; Treatment; Surgical Management; Gastrointestinal tract. 


\section{Diagnóstico de las patologías de tubo digestivo y anexos para proceder al manejo clínico y quirúrgico en el tratamiento adecuado}

Vol. 3, núm. 4., (2019)

Karla Valeria Arcentales Vera; Melissa Mercedes Mendieta Torres; César Enrique Vera Sauhing; Diego Israel Ojeda Maldonado

\section{Introducción.}

Las enfermedades en la vía gastrointestinal superior son muy comunes hoy en día, sobre todo como consecuencia de una mala alimentación y malos hábitos de vida. Las personas con estos padecimientos generalmente no suelen darse cuenta hasta que el trastorno o la enfermedad están avanzados, es decir cuando la enfermedad o trastorno necesitan de intervención médica o incluso quirúrgica. (Ventura, 2016) La palabra endoscopia significa literalmente "mirar al interior". La endoscopia superior permite examinar el recubrimiento de la parte superior del tracto gastrointestinal, que incluye el esófago, estómago y el duodeno. Esta técnica permite la visualización del interior de un órgano hueco o una cavidad corporal, introduciéndolo mediante un agujero natural o una pequeña incisión quirúrgica.

La endoscopia superior ayuda a evaluar los síntomas de dolor abdominal superior, náuseas, vómitos o dificultad para tragar que aparecen en forma persistente. Es la prueba de elección para encontrar la causa de la hemorragia gastrointestinal superior. Además, se puede utilizar la endoscopia superior para obtener biopsias, la cual ayudará a distinguir entre tejidos benignos y malignos. (Ventura, 2016) También, es utilizada para realizar pequeños procedimientos como dilatar zonas estenosadas, extracción de pólipos o tratar el sangrado. Para confirmar una patología del aparato gastrointestinal superior en los pacientes adultos que consultan a una unidad hospitalaria, es necesario el uso de la endoscopia, ya que muchas veces el aparato gastrointestinal se ve expuesto a diversas noxas que pueden poner en riesgo la vida de estos pacientes. 


\section{Diagnóstico de las patologías de tubo digestivo y anexos para proceder al manejo clínico y quirúrgico en el tratamiento adecuado}

Vol. 3, núm. 4., (2019)

Karla Valeria Arcentales Vera; Melissa Mercedes Mendieta Torres; César Enrique Vera Sauhing; Diego Israel Ojeda Maldonado

Entre estas enfermedades se logra señalar, la hemorragia digestiva alta (HDA), la cual sigue siendo una de las complicaciones más graves del aparato digestivo y un motivo frecuente de hospitalización. Las dos causas más habituales son la hemorragia digestiva (HD) por úlcera péptica y la secundaria a hipertensión portal. La hemorragia digestiva baja es menos frecuente que la de origen alto, y tiene tendencia a autolimitarse. Desde el punto de vista de su gravedad, la hemorragia gastrointestinal puede abarcar desde la forma masiva aguda hasta las pérdidas sanguíneas crónicas e intermitentes; pudiendo la hemorragia digestiva alta aguda manifestarse de tres maneras: hematemesis, melena y hematoquezia. (Ventura, 2016).

Estas formas de presentación dependen de la magnitud del sangrado, el cual será evidenciado a través de manifestaciones clínicas del compromiso hemodinámico. Sin embargo, la intensidad y la frecuencia dependen de diversos factores de riesgo como la edad, la presencia de enfermedades concomitantes y el uso de fármacos gastrolesivos que contribuyen incrementando el número de muertes producidas por esta y otras complicaciones, definidas mediante los diferentes métodos y precisión de los diagnósticos aplicados. (Ventura, 2016).

La precisión diagnóstica es definida por Lunney como la aproximación del juicio clínico, mediante la declaración diagnóstica, a los datos que presenta el paciente. La misma incluye la sensibilidad entendida como la probabilidad de que la prueba dé positiva si la condición de estudio está presente (paciente enfermo o con patrón de referencia positivo). (Barret, 2015). Del mismo modo, se puede definir como la proporción de verdaderos positivos respecto al total de enfermos. Por otra parte, se encuentra la especificidad, interpretada como la probabilidad de que la prueba dé negativa si la enfermedad está ausente (paciente sano o con patrón de referencia negativo). 


\section{Diagnóstico de las patologías de tubo digestivo y anexos para proceder al manejo clínico y quirúrgico en el tratamiento adecuado}

Vol. 3, núm. 4., (2019)

Karla Valeria Arcentales Vera; Melissa Mercedes Mendieta Torres; César Enrique Vera Sauhing; Diego Israel Ojeda Maldonado

También se puede definir como la proporción de verdaderos negativos respecto al total de sujetos sanos.

En otro ángulo se encuentra el valor predictivo positivo del diagnóstico, el cual es entendido como la probabilidad de tener la condición de estudio (enfermedad o patrón de referencia positivo) si la prueba ha sido positiva. (Barret, 2015). Igualmente, puede ser definido como la proporción de verdaderos positivos respecto al total de pruebas positivas. Por otra parte, se encuentra el valor predictivo negativo, referido, a través de la probabilidad de no tener la condición de estudio (enfermedad ausente o patrón de referencia negativo) si la prueba ha sido negativa. También puede ser definido como la proporción de verdaderos negativos respecto al total de pruebas negativas.

\section{Métodos y técnicas.}

\section{Fuentes documentales}

El análisis correspondiente a las características que predomina en el tema seleccionado lleva a incluir diferentes fuentes documentales encargadas de darle el respectivo apoyo y en ese sentido cumplir con la valoración de los hechos a fin de generar nuevos criterios que sirven de referencia a otros procesos investigativos. Para (Arias, 2010), las fuentes documentales incorporadas en la investigación documental o bibliográfica, "representa la suma de materiales sistemáticos que son revisados en forma rigurosa y profunda para llegar a un análisis del fenómeno". (p.41). Por lo tanto, se procedió a cumplir con la realización de una lectura previa determinada por encontrar aquellos aspectos estrechamente vinculados con el "DIAGNÓSTICO 


\section{Diagnóstico de las patologías de tubo digestivo y anexos para proceder al manejo clínico y quirúrgico en el tratamiento adecuado}

Vol. 3, núm. 4., (2019)

Karla Valeria Arcentales Vera; Melissa Mercedes Mendieta Torres; César Enrique Vera

Sauhing; Diego Israel Ojeda Maldonado

DE LAS PATOLOGÍAS DE TUBO DIGESTIVO Y ANEXOS PARA PROCEDER A MANEJO

CLÍNICO Y QUIRÚRGICO EN EL TRATAMIENTO ADECUADO" para luego explicar mediante un desarrollo las respectivas apreciaciones generales de importancia.

Técnicas para la Recolección de la Información

La conducción de la investigación para ser realizada en función a las particularidades que determinan a los estudios documentales tiene como fin el desarrollo de un conjunto de acciones encargadas de llevar a la selección de técnicas estrechamente vinculadas con las características del estudio. En tal sentido, (Arias Ob cit) refiere, que es "una técnica particular para aportar ayuda a los procedimientos de selección de las ideas primarias y secundarias”. (p. 71).

Por ello, se procedió a la utilización del subrayado, resúmenes, fichaje, como parte básica para la revisión y selección de los documentos que presentan el contenido teórico. Es decir, que mediante su aplicación de estas técnicas se pudo llegar a recoger informaciones en cuanto a la revisión bibliográfica de los diversos elementos encargados de orientar el proceso de investigación. Tal como lo expresa, (Arias Ob cit) "las técnicas documentales proporcionan las herramientas esenciales y determinantes para responder a los objetivos formulados y llegar a resultados efectivos" (p. 58). Es decir, para responder con eficiencia a las necesidades investigativas, se introdujeron como técnica de recolección el método inductivo, que hizo posible llevar a cabo una valoración de los hechos de forma particular para llegar a la explicación desde una visión general.

Asimismo, se emplearon las técnicas de análisis de información para la realización de la investigación que fue ejecutada bajo la dinámica de aplicar diversos elementos encargados de 


\section{Diagnóstico de las patologías de tubo digestivo y anexos para proceder al manejo clínico y quirúrgico en el tratamiento adecuado}

Vol. 3, núm. 4., (2019)

Karla Valeria Arcentales Vera; Melissa Mercedes Mendieta Torres; César Enrique Vera Sauhing; Diego Israel Ojeda Maldonado

determinar el camino a recorrer por el estudio, según, (Arias, Ob cit) las técnicas de procesamiento de datos en los estudios documentales "son las encargadas de ofrecer al investigador la visión o pasos que debe cumplir durante su ejercicio, cada una de ellas debe estar en correspondencia con el nivel a emplear" (p. 123). Esto indica que, para llevar a cabo el procesamiento de los datos obtenidos, es necesario establecer las técnicas que serán seleccionadas, destacándose en este caso, de manera particular: fichas de resumen, textual, registros descriptivos entre otros, los mismos se deben ajustar al nivel que ha sido seleccionado.

\section{Resultados.}

Patologías del Tubo Digestivo

Se comentan a continuación los hechos fisiopatológicos más destacables y de actualidad, en relación con los grandes cuadros clínicos que afectan al tubo digestivo.

a) Reflujo gastroesofágico: si bien su etiopatogenia puede considerarse de naturaleza multifactorial, parece ser que el factor principal se concreta en la incompetencia del esfínter inferior del esófago, en el sentido de mantener una presión del mismo en reposo anormalmente baja (< $12 \mathrm{~mm} \mathrm{Hg}$, valor normal de referencia). Conviene recordar que es precisamente esta estructura y la presión que ejerce en contra la que constituye la barrera natural antireflujo. Como consecuencia de todo ello el poder agresivo mayor de lo normal, en cantidad y frecuencia, de la secreción y contenido gástricos puede conducir a la génesis de la patología del reflujo (esofagitis, erosiones, etc.). (Barret, 2015). 


\section{Diagnóstico de las patologías de tubo digestivo y anexos para proceder al manejo clínico y quirúrgico en el tratamiento adecuado}

Vol. 3, núm. 4., (2019)

Karla Valeria Arcentales Vera; Melissa Mercedes Mendieta Torres; César Enrique Vera

Sauhing; Diego Israel Ojeda Maldonado

Obviamente pueden existir otros factores que finalmente alteren el gradiente de presión

y faciliten el reflujo, como puede ser el caso entre otros, de la obesidad constitucional o el de la presencia de ciertos alimentos que reducen tal presión (grasas, chocolate, menta, etc.), aunque en esencia el mecanismo fisiopatológico sigue siendo el mismo.

b) Ulcera péptica: en este apartado puede significarse que, desde la edición anterior, se ha producido un avance sustancial. Aun teniendo por válida la teoría fisiopatológica del desequilibrio de Alquist, entre factores agresivos y defensivos, hoy parece claramente establecido que, dejando al margen la patología ulcerosa inducida por fármacos AINE, o la coexistente dentro de un síndrome de Zollinger-Ellison, en el desajuste de estos últimos (defensivos) interviene decisivamente la infección por Helicobacter pylori, de modo que ya no se concibe el control y la curación definitiva de una úlcera péptica, si no es mediante el correspondiente tratamiento antimicrobiano, capaz de erradicar al microrganismo que se refiere. (Barret, 2015).

De acuerdo con datos de la bibliografía (5-7), excluidos los procesos ulcerosos antes citados, el 80- $95 \%$ de los casos de úlcera duodenal y el $80 \%$ de los de úlcera gástrica se asocian definitivamente con la existencia del Helicobacter pylori. Se han documentado varios efectos de este germen que supone una alteración del concepto defensivo de "barrera-mucosa" (reacción inflamatoria que cambia su arquitectura, desintegración del moco por acción de proteasas específicas, disminución de la secreción duodenal de bicarbonato, etc.). (Barret, 2015)

c) Dispepsia funcional y síndrome del intestino irritable: etimológicamente, para definir el término de dispepsia habría que referirse a la existencia de molestias por mala o difícil (dis) digestión (pepsia o pepto). Por tanto, se incluirían aquellos procesos de malestar abdominal, con 


\section{Diagnóstico de las patologías de tubo digestivo y anexos para proceder al manejo clínico y quirúrgico en el tratamiento adecuado}

Vol. 3, núm. 4., (2019)

Karla Valeria Arcentales Vera; Melissa Mercedes Mendieta Torres; César Enrique Vera Sauhing; Diego Israel Ojeda Maldonado

sintomatología muy diversa e imprecisa de origen funcional, es decir, una vez eliminadas otras múltiples causas orgánicas o estructurales que pudiesen justificarlas (reflujo, úlcera, trastornos pancreáticos o hepáticos, enfermedades metabólicas, etc.). (Barret, 2015) En tal concepto podría admitirse también el de síndrome de intestino irritable (o más específicamente, de colon irritable) en el que se refiere un dolor abdominal anodino, acompañado de otros síntomas, sin que se identifique tampoco un origen orgánico definido.

Desde el punto de vista fisiopatológico cabe señalar que su conocimiento es poco claro y más bien escaso, pudiendo intervenir múltiples factores, aunque parece, sobre todo por lo que respecta al síndrome del Intestino o Colon Irritable, que se consolida cada vez más la hipótesis de la existencia de una hipersensibilidad individual del intestino (con percepción aumentada y dolorosa), ante sensaciones o estímulos de plenitud o distensión, a lo largo del aparato digestivo. (Barret, 2015). En esta línea de pensamiento pierde valor la importancia que se concedía anteriormente a los factores psicosociales o de trastornos afectivos, aunque no pueda desestimarse del todo la interrelación entre ambos hechos, como expresión final de carácter psicosomático.

d) Náuseas y vómitos: se comentan brevemente dos situaciones de náuseas y vómitos que interesan particularmente a los Servicios Farmacéuticos en el medio hospitalario, dada la importancia fisiopatológica que adquiere el mecanismo de su producción, a través de la vía neurotransmisora serotoninérgica (receptores 5-HT3), y la notable repercusión que ello supone en la estrategia de su prevención o tratamiento farmacológicos. (Barret, 2015). La émesis inducida por los fármacos antineoplásicos depende en su severidad e incidencia de ciertas variables, entre las que destaca el propio potencial emetógeno de la molécula o moléculas que componen el 


\section{Diagnóstico de las patologías de tubo digestivo y anexos para proceder al manejo clínico y quirúrgico en el tratamiento adecuado}

Vol. 3, núm. 4., (2019)

Karla Valeria Arcentales Vera; Melissa Mercedes Mendieta Torres; César Enrique Vera

Sauhing; Diego Israel Ojeda Maldonado

régimen quimioterápico, y se relaciona en su etiopatogenia con la acción excitadora de la zona quimiorreceptora gatillo (ZQG) de tales principios activos o de sus metabolitos, mediada por el sistema transmisor comentado de serotonina

Otro tanto puede señalarse con relación a las náuseas y vómitos que ocurren tras la cirugía, como hecho patológico principal del periodo postoperatorio, que afecta al 25-30\% de los casos. Por diversos mecanismos las acciones de la propia cirugía, anestesia, etc., inciden por la vía serotoninérgica ya mencionada, tanto sobre el tracto gastrointestinal como sobre la ZQG, provocando la situación emética que se comenta. (Barret, 2015). Más adelante, en el apartado correspondiente, se discutir el valor terapéutico para estas circunstancias patológicas de los fármacos antagonistas serotoninérgicos de tipo 5- HT3, como substancias de reciente incorporación a la terapéutica, con un excelente perfil de seguridad y eficacia a estos fines.

e) Alteraciones intestinales. Diarrea y estreñimiento: por lo que se refiere a la fisiopatología de la diarrea, se recogen seguidamente los principales mecanismos de su producción que conviene conocer, independientemente de las múltiples causas etiológicas que pueden quedar representadas en cada uno de ellos:

- Alteración en la absorción intestinal, de forma que solutos retenidos en la luz generen un efecto osmótico de arrastre. En este grupo habría que incluir todo el grupo de los cuadros diarreicos relacionados con los síndromes de malabsorción (enfermedad celíaca y de disacaridasas, síndrome de intestino corto, yatrogenia postgastrectomía, otras causas de esteatorrea, etc.). 


\section{Diagnóstico de las patologías de tubo digestivo y anexos para proceder al manejo clínico y quirúrgico en el tratamiento adecuado}

Vol. 3, núm. 4., (2019)

Karla Valeria Arcentales Vera; Melissa Mercedes Mendieta Torres; César Enrique Vera Sauhing; Diego Israel Ojeda Maldonado

- Incremento neto de los procesos de secreción intestinal, por agresión de carácter inflamatorio sobre el epitelio de la mucosa (diarrea secretora, con eliminación en exceso de agua y electrolitos). Se agrupan en este apartado los procesos exudativos y secretores de las enfermedades inflamatorias crónicas del intestino, comentadas en el apartado siguiente, y sobre todo la diarrea aguda de origen y complicación más grave y directa, como es la deshidratación.

- Trastornos de naturaleza motora, que incluye el resto de los síndromes diarreicos, una vez eliminados los anteriores, y que suele originarse más comúnmente por aumento en el peristaltismo propulsor, como en el síndrome del intestino irritable, pero también por disminución de éste que, al permitir un sobrecrecimiento microbiano, conduce a una situación de malabsorción con esteatorrea y diarrea.

- Con respecto al estreñimiento conviene recordar que el hecho patológico más significativo lo representa el estreñimiento crónico idiopático, es decir, de causa desconocida, descartados los cuadros de estreñimiento que son síntoma de una enfermedad orgánica gastrointestinal, o incluso secundarios a enfermedad sistémica metabólica o endocrina. En este caso la fisiopatología hace referencia, o bien a un trastorno en la motilidad colónica, tanto de hipomotilidad por reducción de los movimientos propulsivos, como de hipermotilidad con incremento de los no propulsivos o segmentarios, o bien a un cuadro de obstrucción mecánica o funcional de la parte distal de salida colon-recto. (Barret, 2015).

f) Enfermedades inflamatorias crónicas del intestino: como avance más significativo en los últimos tiempos, aunque sigue siendo cierto que la fisiopatología precisa de tales enfermedades 


\section{Diagnóstico de las patologías de tubo digestivo y anexos para proceder al manejo clínico y quirúrgico en el tratamiento adecuado}

Vol. 3, núm. 4., (2019)

Karla Valeria Arcentales Vera; Melissa Mercedes Mendieta Torres; César Enrique Vera

Sauhing; Diego Israel Ojeda Maldonado

no ha sido todavía desentrañada, se destaca que parece afianzarse cada vez con mayor evidencia, el origen inmunológico de ellas, no sólo de la Colitis Ulcerosa, sino también y más recientemente de la propia Enfermedad de Crohn. (Barret, 2015).

Hoy día, está bien documentado que por ejemplo en las crisis agudas de Colitis Ulcerosa hay un aumento significativo en la producción por parte de la mucosa afectada, de diversos elementos inmunológicos (células T, macrófagos, moléculas de IgG, etc.). De igual modo se constata que en la enfermedad activa de Crohn existe una importante participación de neutrófilos, células T, macrófagos, etc., pero particularmente citocinas proinflamatorias como interleucinas y sobre todo el factor de necrosis tumoral alfa (FNTa) que se considera clave para iniciar y perpetuar el proceso patológico de inflamación de la mucosa intestinal (9). Tanto es así que hoy constituye una diana proritaria en las estrategias terapéuticas en fase de investigación.

\section{Tubo Digestivo}

La hemorragia digestiva alta (HDA) que consiste en la pérdida de sangre hacia la luz del tubo digestivo en una zona comprendida entre el esfínter esofágico superior y el ángulo de Treitz constituye un motivo frecuente de consulta. Este sangrado puede proceder de órganos digestivos o de estructuras adyacentes que vierten su contenido en esta localización, por conductos naturales (árbol biliopancreático) o patológicos (fístulas espontáneas, traumáticas o quirúrgicas). (Comte, 2016). Se manifiesta clínicamente por hematemesis, melena y otros tipos de sintomatología, independientemente de la lesión de origen, diferenciándose sólo por su magnitud, evidenciable a través de la presencia o ausencia de compromiso hemodinámico y magnitud del mismo. 


\section{Diagnóstico de las patologías de tubo digestivo y anexos para proceder al manejo clínico y quirúrgico en el tratamiento adecuado}

Vol. 3, núm. 4., (2019)

Karla Valeria Arcentales Vera; Melissa Mercedes Mendieta Torres; César Enrique Vera Sauhing; Diego Israel Ojeda Maldonado

En principio, toda HDA franca debe considerarse como potencialmente grave, y por lo tanto, merecedora de esfuerzos diagnósticos y terapéuticos intensos. La morbilidad y mortalidad de los pacientes se relaciona con ciertos factores de riesgos, como la edad, enfermedades asociadas magnitud de la hemorragia, origen de la misma, alcoholismo, ingesta de medicamentos, etc. (Comte, 2016). Otros factores principales que influyen en la aparición de HDA son la infección por Helicobacter pylori y la administración de antiinflamatorios no esteroideos. Las lesiones agudas de la mucosa gástrica constituyen el $20 \%$ de las causas de HDA y se asocian a la toma de AINE, alcohol, o estado crítico del enfermo con ventilación mecánica, 10 coagulopatía, lesiones neurológicas graves, politraumatizados, etc.

La esofagitis, síndrome de Mallory-Weiss, lesiones vasculares, neoplasias esofagogástricas, fístula aortoentérica y hemobilia son causas menos frecuentes. Los procedimientos diagnósticos disponibles son la anamnesis y exploración física, hemograma, bioquímica, estudio de coagulación, colocación de sonda nasogástrica y lavados, panendoscopia oral, radiología baritada, angiografía, gammagrafía con sulfuro coloidal o hematíes marcados y la laparotomía exploradora. (Comte, 2016).

La esofagogastroduodenoscopía tiene un papel decisivo en la evaluación diagnóstica y manejo terapéutico de la HDA. La seguridad, refinamiento anatómico, y su capacidad para obtener muestras de tejido soportan su uso como principal instrumento diagnóstico. La amplia diversidad de instrumentos endoscópicos disponibles permite ampliar su aplicación diagnóstica y terapéutica a un amplio rango de patologías, tanto benignas como neoplásicas, precisando de esta manera la 


\section{Diagnóstico de las patologías de tubo digestivo y anexos para proceder al manejo clínico y quirúrgico en el tratamiento adecuado}

Vol. 3, núm. 4., (2019)

Karla Valeria Arcentales Vera; Melissa Mercedes Mendieta Torres; César Enrique Vera Sauhing; Diego Israel Ojeda Maldonado

causa y el origen de la hemorragia en la mayoría de los casos (90 - 95\%), con una incidencia de complicaciones menor del $0,01 \%$.

Desde esta perspectiva se logra entender que la hemorragia digestiva es causa importante de morbilidad y mortalidad en el mundo, cuyas incidencias son difíciles de determinar a pesar de la gran cantidad de reportes que se encuentra de la literatura médica. Estudios poblacionales de las décadas del 60 y 70 estiman tasas anuales de hemorragia digestiva alta que van desde 48 a 144 episodios por 100,000 habitantes. En los Estados Unidos de Norteamérica es responsable de cerca de 300,000 admisiones al año. Estudios recientes sugieren que estas cifras no reflejan la realidad ya que no consideran las hemorragias digestivas que se producen durante la hospitalización; las cuales podrían alcanzar hasta el 50\% de los episodios de hemorragia digestiva. (Comte, 2016).

Su etiología es variable, siendo las causas más frecuentes de hemorragia digestiva alta la enfermedad ulcero péptica que representa el 50\% de los casos, seguida de las lesiones agudas de mucosa digestiva, las várices esofágicas y por último el síndrome de Mallory Weiss. La mortalidad debido a hemorragia digestiva alta no ha variado en los últimos 50 años y permanece entre 8 a 10\%, a pesar de los avances en la terapia farmacológica, intervención endoscópica y manejo en unidades de cuidado intensivo, lo que se atribuiría a la cada vez mayor proporción de pacientes mayores de 60 años, muchos de los cuales se presentan con otras enfermedades concomitantes. (Comte, 2016).

Sin embargo, en los países occidentales la incidencia de Hemorragia Digestiva Alta es de 100 a 150 casos por 100,000 habitantes al año; con una mortalidad del 10 al 14\%; constituyendo 


\section{Diagnóstico de las patologías de tubo digestivo y anexos para proceder al manejo clínico y quirúrgico en el tratamiento adecuado}

Vol. 3, núm. 4., (2019)

Karla Valeria Arcentales Vera; Melissa Mercedes Mendieta Torres; César Enrique Vera Sauhing; Diego Israel Ojeda Maldonado

la principal urgencia gastroenterológica. Desde el punto de vista de su gravedad, la hemorragia gastrointestinal puede abarcar desde la forma masiva aguda hasta las pérdidas sanguíneas crónicas e intermitentes; pudiendo la hemorragia digestiva alta aguda manifestarse de tres maneras: hematemesis, melena y hematoquecia; estas formas de presentación dependen de la magnitud del sangrado, evidenciado a través de manifestaciones clínicas del compromiso hemodinámica. (Comte, 2016).

\section{Factores endoscópicos con carácter Pronóstico}

Los parámetros endoscópicos que tienen valor predictivo sobre la evolución de la hemorragia, especialmente en los pacientes con HD secundaria a úlcera, son los siguientes: ) Sangrado activo arterial, en chorro o babeante (frecuencia de recidiva hemorrágica del $85 \%$ ). (Rentnz, 2017)

Vaso visible no sangrante (protuberancia pigmentada, roja, azul o púrpura, situada en el fondo del cráter ulceroso). Se trata de la propia arteria que hace prominencia, de un pseudoaneurisma arterial o de un coágulo rojo taponando el vaso arterial (frecuencia de recidiva hemorrágica del 35-55 \%). Coágulo rojo taponando la lesión (frecuencia de recidiva hemorrágica $\operatorname{del} 25 \%)$.

\section{Métodos de Diagnóstico}

El método diagnóstico habitual es la endoscopia alta y con menor frecuencia la arteriografía, la enteros copia, la gammagrafía y la endoscopia peroperatoria. 


\section{Diagnóstico de las patologías de tubo digestivo y anexos para proceder al manejo clínico y quirúrgico en el tratamiento adecuado}

Vol. 3, núm. 4., (2019)

Karla Valeria Arcentales Vera; Melissa Mercedes Mendieta Torres; César Enrique Vera

Sauhing; Diego Israel Ojeda Maldonado

Arteriografía. La indicación de la arteriografía se halla limitada a aquellos pacientes

con HDA que presentan una hemorragia persistente y no ha podido localizarse su origen por endoscopia alta o baja, y que, por su gravedad, resulta necesario llegar a un diagnóstico de la lesión. Para que esta técnica sea rentable, desde un punto de vista diagnóstico, es condición imprescindible que al inyectar el contraste se esté produciendo una extravasación sanguínea activa en una cantidad mínima de $0,5 \mathrm{ml} / \mathrm{min}$. Rentnz, 2017).

La arteriografía, además de su valor diagnóstico, también puede tener una utilidad terapéutica, actuando sobre la lesión sangrante; para ello se debe practicar primero una arteriografía selectiva y una vez localizado el vaso sangrante se procede a la embolización de éste mediante gelfoam o esponja de gelatina. Una indicación clara de esta técnica es el caso de hemobilia, aparecida como complicación de una biopsia hepática. Rentnz, 2017=.

Enteroscopía. Se realizará cuando se haya descartado el origen gastroduodenal por gastroscopía y del colon por colonoscopia. Es una técnica delicada y precisa de sedación del paciente. Además, nos permitirá visualizar el intestino delgado. Gammagrafía marcada con tecnecio En esta exploración la acumulación del radioisótopo en el lugar de la hemorragia puede ser detectada por el contador gamma. Rentnz, 2017).

Se utilizará en aquellos casos de HD de origen no aclarado. Gastroscopía Deberá practicarse a todos los pacientes con HDA confirmada por la presencia de hematemesis y/o melenas o salida de sangre por la sonda nasogástrica. Se procurará realizarla precozmente, una vez se haya logrado remontar al paciente y cuando éste se encuentre estable hemodinámicamente. Siempre que sea posible se efectuará con sedación del paciente. La realización de una endoscopia 


\section{Diagnóstico de las patologías de tubo digestivo y anexos para proceder al manejo clínico y quirúrgico en el tratamiento adecuado}

Vol. 3, núm. 4., (2019)

Karla Valeria Arcentales Vera; Melissa Mercedes Mendieta Torres; César Enrique Vera Sauhing; Diego Israel Ojeda Maldonado

urgente y el momento preciso para llevarla a cabo se sopesarán cuidadosamente en pacientes con síndrome de deprivación alcohólica o enfermedades asociadas graves, tales como insuficiencia respiratoria, cardíaca o de miocardio reciente. El informe endoscópico deberá describir: tipo, tamaño y localización de las lesiones; estigmas de hemorragia reciente; presencia o ausencia de sangre fresca o digerida en el tracto digestivo explorado Rentnz, 2017).

Tabla 1. Estigmas endoscópicos de Hemorragia Reciente

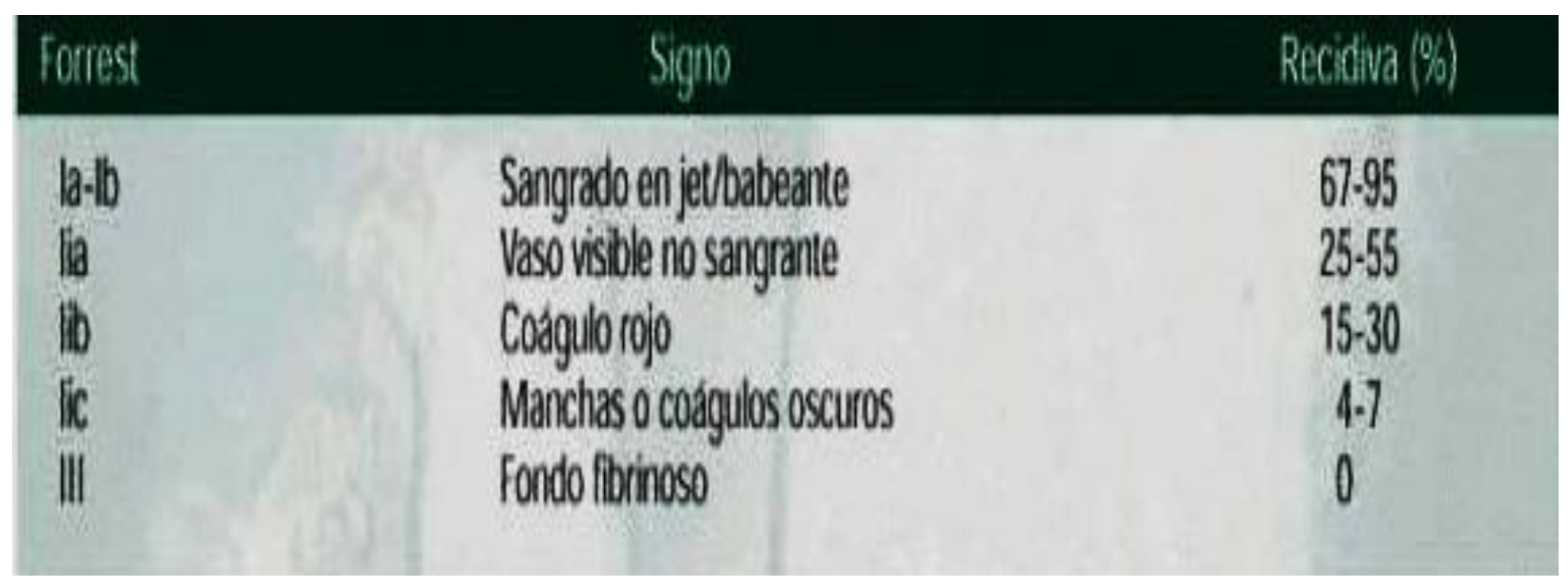

Fuente: Rentnz, 2017).

La gastroscopía urgente debe realizarse en el plazo más breve posible, ya que existe una correlación directa entre la eficacia del diagnóstico endoscópico y el tiempo transcurrido desde el inicio de la hemorragia. Rentnz, 2017).

La endoscopía digestiva alta es un elemento de gran utilidad en el manejo de los pacientes que tienen una hemorragia digestiva. Sin embargo, el mejor momento para realizarla debe ser definido clínicamente de modo de obtener no solamente la posibilidad de un adecuado diagnóstico, 


\section{Diagnóstico de las patologías de tubo digestivo y anexos para proceder al manejo clínico y quirúrgico en el tratamiento adecuado}

Vol. 3, núm. 4., (2019)

Karla Valeria Arcentales Vera; Melissa Mercedes Mendieta Torres; César Enrique Vera

Sauhing; Diego Israel Ojeda Maldonado

sino también una adecuada acción terapéutica si está indicada. Es necesario tener presente que entre el 70 y el $80 \%$ de los pacientes que tienen una hemorragia digestiva alta de causa no variceal, se detiene espontáneamente. Rentnz, 2017).

Por lo tanto, el paciente debe ser ingresado en una unidad de cuidados intermedios y monitorizado cuidadosamente; si en las próximas horas se observa que el paciente se estabiliza hemodinámicamente, será muy útil darle agua por boca, de manera que lave su estómago y la endoscopía podrá ser realizada dentro de las próximas 8 ó 12 hrs, si por el contrario pese a las medidas de reanimación el paciente se mantiene inestable hemodinámicamente, dependiendo de volumen, con tendencia a la hipotensión o presenta sangrado activo externo (nueva hematemesis o sangre fresca por el recto), unido a inestabilidad hemodinámica, ese paciente requerirá una endoscopía inmediata. Rentnz, 2017).

\section{Valoración Pronóstica}

Tanto antes como después de la endoscopia se debe realizar una valoración pronostica. Como instrumento de soporte para la estratificación se recomienda el uso de escalas pronósticas. Las más conocidas son las de Rockall y la de Blatchford. La escala de Blatchford utiliza datos clínicos y de laboratorio, mientras que la escala de Rockall incluye también variables endoscópicas. Una puntuación de cero en la escala de Blatchford permite identificar un pequeño subgrupo de pacientes de muy bajo riesgo que podrían ser dados de alta incluso sin necesidad de realizar gastroscopia urgente. (Carret, 2015). 


\section{Diagnóstico de las patologías de tubo digestivo y anexos para proceder al manejo clínico y quirúrgico en el tratamiento adecuado}

Vol. 3, núm. 4., (2019)

Karla Valeria Arcentales Vera; Melissa Mercedes Mendieta Torres; César Enrique Vera Sauhing; Diego Israel Ojeda Maldonado

En relación con el estado hemodinámico del paciente es importante mantener vigilancia de signos vitales, los cuales nos permiten estimar las perdidas hemáticas y clasificar al paciente de acuerdo a las alteraciones de los signos vitales, por lo que es recomendable el uso de la clasificación de la hemorragia. Tras la endoscopia los pacientes de bajo riesgo de recidiva pueden ser dados de alta inmediatamente si cumplen los criterios adecuados. El alta hospitalaria temprana en estos pacientes reduce significativamente los costes y no aumenta las complicaciones ni la mortalidad. (Carret, 2015).

Los criterios para el alta precoz son: úlcera de base limpia o mancha de hematina, estabilidad hemodinámica, ausencia de enfermedades graves, fácil acceso al hospital y adecuado apoyo familiar en el domicilio del paciente. Por otro lado, es necesario ingresar a los pacientes de mayor gravedad que son fundamentalmente aquellos con estigmas endoscópicos de alto riesgo que han requerido tratamiento endoscópico. Dado que la mayor parte de recidivas acontecen durante este período, se recomienda un ingreso mínimo de 72 horas. (Carret, 2015).

Debe valorarse el ingreso de los pacientes con sangrados más graves en unidades de cuidados intensivos o unidades de sangrantes, en especial si presentan inestabilidad hemodinámica y/o sangrado activa persistentes. (Carret, 2015) Los factores que permiten identificar a los pacientes de alto riesgo de recidiva y mortalidad tras el tratamiento endoscópico son:

- Clínicos: edad avanzada, comorbilidad grave y shock hipovolémico

- Endoscópicos: causa del sangrado (la úlcera péptica presenta un peor pronóstico que las erosiones gastroduodenales o el síndrome de Mallory-Weiss), úlceras de tamaño superior 


\section{Diagnóstico de las patologías de tubo digestivo y anexos para proceder al manejo clínico y quirúrgico en el tratamiento adecuado}

Vol. 3, núm. 4., (2019)

Karla Valeria Arcentales Vera; Melissa Mercedes Mendieta Torres; César Enrique Vera Sauhing; Diego Israel Ojeda Maldonado

a $2 \mathrm{~cm}$, hemorragia activa en al momento de la endoscopia, especialmente hemorragia pulsátil y la localización en la cara posterior de bulbo o porción alta de la curvatura menor gástrica.

La probabilidad de recidiva hemorrágica puede predecirse a partir de los estigmas endoscópicos de alto riesgo descritos por Forrest. Así, en pacientes con sangrado activo y en aquellos con vaso visible no sangrante (Forrest I y IIa, respectivamente) el tratamiento endoscópico reduce significativamente la probabilidad de recidiva, la necesidad de cirugía urgente y la mortalidad. En caso de coágulo adherido (Forrest IIb) se recomienda lavado vigoroso y realizar tratamiento en función de la lesión subyacente. (Carret, 2015).

Si no se desprende el coágulo, se recomienda tratamiento mediante inyección a su través. Finalmente, ante una úlcera limpia o con mancha de hematina (Forrest III) no está indicado tratamiento endoscópico ya que la probabilidad de recidiva es muy baja $(<5,0 \%) 13$. La utilización de un second-look endoscópico a las 12-24 horas de la endoscopia inicial puede ser útil en pacientes con alto riesgo de recidiva tras el tratamiento endoscópico. Sin embargo, su uso sistemático no resulta coste-efectivo, por lo que debe restringirse a pacientes seleccionados. (Carret, 2015).

Sangrado Digestivo Alto SDA

El sangrado digestivo alto (SDA) se define como la presencia de sangre en el tracto digestivo alto, proveniente de la mucosa o vasos sanguíneos que se localizan entre el esófago y el ángulo de Treitz. Las principales manifestaciones clínicas asociadas al SDA están dadas por la 


\section{Diagnóstico de las patologías de tubo digestivo y anexos para proceder al manejo clínico y quirúrgico en el tratamiento adecuado}

Vol. 3, núm. 4., (2019)

Karla Valeria Arcentales Vera; Melissa Mercedes Mendieta Torres; César Enrique Vera Sauhing; Diego Israel Ojeda Maldonado

presencia de hematemesis, melena y en algunos casos hematoquezia, esta última relacionada con sangrados que cursan con aceleración del tránsito intestinal. (Carret, 2015).

Esta patología es un motivo frecuente de consulta en urgencias, que implica además un importante número de ingresos tanto al área crítica de Urgencias, Unidad de Cuidados Intensivos y hospitalización. La mortalidad por esta causa es variable, se estima en 3,5\% al 10,0\% y esta depende sobre todo de la edad del paciente y sus posibles enfermedades asociadas, la cuantía del sangrado y los hallazgos que se encuentren durante la realización del procedimiento endoscópico. (Carret, 2015).

El sangrado digestivo alto es autolimitado o cesa espontáneamente en el 80,0\% de los casos. La causa más frecuente es la hemorragia por úlcera péptica (25,0\% ulcera duodenal-23,0\% ulcera gástrica). Con este protocolo clínico buscamos normar el manejo hospitalario de los pacientes que ingresan al área de Urgencias con el fin de unificar criterios que conlleven al manejo adecuado de los pacientes con sangrado digestivo alto. (Carret, 2015).

Este protocolo clínico es un instrumento normativo, de orientación eminentemente de práctica médica, en el que se busca unificar criterios y tener como resultado adecuado el manejo del paciente con sangrado digestivo alto; va dirigido a Médicos Gastroenterólogos, Endoscopistas, Emergenciólogos, Intensivistas, Médicos de Familia y de Atención Primaria, Imagenólogos Intervencionistas y Cirujanos Generales de las diferentes unidades médicas del Sistema Nacional de Salud. (Carret, 2015). 


\section{Diagnóstico de las patologías de tubo digestivo y anexos para proceder al manejo clínico y quirúrgico en el tratamiento adecuado}

Vol. 3, núm. 4., (2019)

Karla Valeria Arcentales Vera; Melissa Mercedes Mendieta Torres; César Enrique Vera

Sauhing; Diego Israel Ojeda Maldonado

En términos generales, el sangrado digestivo alto representa la pérdida sanguínea situada por encima del ligamento de Treitz; Clínicamente se manifiesta en forma de hematemesis de sangre fresca o en «poso de café», de melena o, con menor frecuencia, como hematoquezia. El sangrado digestivo alto (SDA) tiene una incidencia aproximada de 50 a 150 cada 100.000 habitantes/año, presenta una mortalidad del $3,5 \%$ al $10,0 \%$, y de $33,0 \%$ en los pacientes hospitalizados por otra razón. La hemorragia digestiva aguda constituye un problema médico que conlleva una significativa morbilidad, mortalidad y un elevado consumo de recursos sanitarios. (Carret, 2015).

Melena:

Se define como la deposición de heces negras, "pegajosas", "alquitranadas" y malolientes que hasta un 95,0\% constituyen un sangrado digestivo alto (SDA) que indica una extravasación de sangre procedente de tramos del tubo digestivo proximales al ángulo de Treitz. En el resto la sangre procede de tramos más distales (intestino delgado o colon derecho). (Salón, 2017).

Hematemesis:

Significa vómitos de sangre que puede ser de color rojizo si es reciente y negro o en poso de café cuando es sangre digerida. (Salón, 2017).

Sangrado Digestivo Oculto: 


\section{Diagnóstico de las patologías de tubo digestivo y anexos para proceder al manejo clínico y quirúrgico en el tratamiento adecuado}

Vol. 3, núm. 4., (2019)

Karla Valeria Arcentales Vera; Melissa Mercedes Mendieta Torres; César Enrique Vera Sauhing; Diego Israel Ojeda Maldonado

El sangrado digestivo oculto (SDO) hace referencia a la presentación inicial de un test de sangre oculta en heces ( $\mathrm{SOH}$ ) positivo y/o anemia ferropénica (AF), sin evidencia de sangre fecal visible por el paciente o el médico, mientras que el sangrado digestivo de origen incierto (SDI) es aquél que persiste o recurre (por ejemplo, test de $\mathrm{SOH}$ o AF recurrente o persistente o sangrado visible) tras un estudio inicial endoscópico alto o colonoscopia. (Salón, 2017).

\section{Arteriografía:}

Se encuentra limitada a aquellos pacientes con sangrado digestivo alto persistente y no ha podido localizarse su origen por endoscopia alta o baja, y que, por su gravedad, resulta necesario llegar a un diagnóstico de la lesión. Detecta una cantidad mínima de extravasación de sangre de $0.5 \mathrm{ml} / \mathrm{min}$. También puede tener utilidad terapéutica actuando sobre la lesión sangrante. Entre las complicaciones de la embolización la más grave es la isquemia intestinal. (Salón, 2017)

\section{Cápsula Endoscópica:}

Tiene un mayor rendimiento diagnóstico como técnica alternativa en el sangrado digestivo de origen oscuro. En el sangrado digestivo agudo está indicada cuando la gastroscopia y la colonoscopia son normales, juntamente con la arteriografía o la angiotomografia, y previa enteroscopía. (Salón, 2017).

\section{Enteroscopía:}




\section{Diagnóstico de las patologías de tubo digestivo y anexos para proceder al manejo clínico y quirúrgico en el tratamiento adecuado}

Vol. 3, núm. 4., (2019)

Karla Valeria Arcentales Vera; Melissa Mercedes Mendieta Torres; César Enrique Vera Sauhing; Diego Israel Ojeda Maldonado

Se realizará cuando se haya descartado el origen gastroduodenal por gastroscopia y del colon por colonoscopia. Es una técnica delicada, requiere sedar al paciente y nos permitirá visualizar el intestino delgado más allá del ángulo de Treitz. Y en caso de hallar una lesión sangrante realizar un tratamiento endoscópico. (Salón, 2017).

\section{Gammagrafía marcada con Tecnecio 99}

Utiliza hematíes marcados con tecnecio 99; la acumulación del radioisótopo en el lugar de la hemorragia puede ser detectada con el contador gamma. Es capaz de detectar sangrados con extravasación baja $0.1 \mathrm{ml} / \mathrm{min}$, con una capacidad de diagnóstico que varía del 50,0-70,0\%. Se utilizará en aquellos casos de sangrado digestivo de origen no aclarado. (Salón, 2017)

\section{Tratamiento}

Tratamiento inicial. Es primordial realizar una historia clínica del paciente ingresado con HD y evaluar su estado hemodinámico (presión arterial, pulso, diuresis), así como conocer la existencia de enfermedades asociadas graves y, muy especialmente, de enfermedad hepática (ictericia, ascitis, encefalopatía). También, hay que preguntar sobre las características del sangrado: hematemesis y/o melenas. Se debe inquirir sobre el antecedente de ingesta previa de fármacos, especialmente AINE, anticoagulantes e ingesta de alcohol. (Salón, 2017).

La restauración de la volemia es el objetivo inmediato en toda hemorragia, prioritario a la recuperación de la anemia. Para ello, se administrarán en el plazo más breve fluidos por vía intravenosa, cuya cantidad y tipo se decidirán en función de la situación del paciente. Con frecuencia debe hacerse incluso antes de la realización de la historia clínica detallada. Mientras se 


\section{Diagnóstico de las patologías de tubo digestivo y anexos para proceder al manejo clínico y quirúrgico en el tratamiento adecuado}

Vol. 3, núm. 4., (2019)

Karla Valeria Arcentales Vera; Melissa Mercedes Mendieta Torres; César Enrique Vera Sauhing; Diego Israel Ojeda Maldonado

efectúan las pruebas de compatibilidad sanguínea previas a la administración de concentrados de hematíes $(\mathrm{CH})$, se pasarán soluciones cristaloides, si la situación hemodinámica del paciente lo requiere. (Salón, 2017).

Estará indicado ante toda hemorragia importante colocar una vía venosa central y sonda vesical. Se monitorizará al paciente, realizando controles frecuentes de presión arterial, frecuencia cardíaca y respiratoria, presión venosa central, saturación de oxígeno y diuresis horaria. La cantidad de unidades de $\mathrm{CH}$ a transfundir estará en función de las pérdidas estimadas, de la persistencia de la hemorragia y de la situación general del enfermo. Se deben mantener unas cifras de hemoglobina superiores a 8 g/dl. (Salón, 2017).

La administración de plasma o plaquetas estará indicada únicamente cuando se detecte un trastorno grave de la coagulación, lo cual ocurre en contadas ocasiones, excepto en los pacientes que toman anticoagulantes. Los pacientes con hemorragia grave o riesgo probable de recidiva hemorrágica deben permanecer ingresados en unidades de críticos o de sangrantes. Cuando se sospecha una HDA, o en caso de duda, se deberá colocar una sonda nasogástrica y, en caso de detectarse sangre oscura o roja, se realizarán lavados gástricos periódicos con el fin de conocer la evolución de la hemorragia y como preparación previa a la práctica de la gastroscopia.

Tratamiento endoscópico. Suele considerarse el método más eficaz para el control de la hemorragia ulcerosa aguda y según el aspecto de ella, la prevención de una hemorragia recurrente conforme lo demostró desde hace más de una década Cook en 1992. Reduce la mortalidad, sobre todo en pacientes de alto riesgo como lo ha señalado, la American Society for Gastrointestinal Endoscopy. A pesar de que en manos inexpertas las complicaciones, como agravar la hemorragia 


\section{Diagnóstico de las patologías de tubo digestivo y anexos para proceder al manejo clínico y quirúrgico en el tratamiento adecuado}

Vol. 3, núm. 4., (2019)

Karla Valeria Arcentales Vera; Melissa Mercedes Mendieta Torres; César Enrique Vera

Sauhing; Diego Israel Ojeda Maldonado

o provocar perforaciones puede llegar hasta el 20\%, en servicios especializados estas complicaciones son raras y se sitúan, en o en menos del $1 \%$. Entre estos procedimientos debe consignarse en orden de posibilidades:

- Terapia con inyecciones que representa la principal modalidad no térmica, utilizándose adrenalina (1:10 000) etanol absoluto, dextrosa al 50\% en agua y aun solución fisiológica.

- La ejecución de abones alrededor de la lesión, es la causa fundamental para detener el sangrado activo de un vaso como lo señala también Cook.

- La electrocoagulación monopolar no es la más adecuada, por la adherencia del tejido; en la zona donde se ha producido la electricidad; mejor resultado da la electrocoagulación bipolar o multipolar, pero con la misma atingencia.

- Sonda térmica, llamada también probeta caliente, con su extremo cubierto de un material de teflón impide la adherencia del tejido y el éxito de este procedimiento está en la aplicación directa con la sonda para obtener el taponamiento del vaso sangrante y el uso de una corriente de calor medido en Jules y limpieza de la zona comprometida con la ayuda de un chorro de agua regulado automáticamente, también posibilita aplicaciones repetidas obteniéndose mejores resultados.

- La aplicación de rayo láser es procedimiento caro, poco práctico para detener el sangrado sustituible por otros métodos. Algunas unidades especializadas cuentan con argón plasma, que por su fácil manejo es de mucha utilidad para lesiones difusas, como es el caso de la gastropatía hipertensiva sangrante. 


\section{Diagnóstico de las patologías de tubo digestivo y anexos para proceder al manejo clínico y quirúrgico en el tratamiento adecuado}

Vol. 3, núm. 4., (2019)

Karla Valeria Arcentales Vera; Melissa Mercedes Mendieta Torres; César Enrique Vera Sauhing; Diego Israel Ojeda Maldonado

- La probeta caliente y aun la inyecto terapia son procedimientos que dan mejor resultado, en caso de sangrado arterial, mientras que el argón plasma es más efectivo en las lesiones difusas ya mencionadas.

- Las bandas elásticas son útiles en caso de hemorragia en el estómago por lesión de Dieulafoy. Su aplicación será comentada en la hemorragia váriceal.

- El tratamiento con láser o hemoclips da resultados iguales o ligeramente superiores a los obtenidos con las otras modalidades, pero las sondas térmicas o bipolares y las inyecciones, de prácticamente cualquier sustancia, son los métodos preferidos debido a su menor complejidad, asequibilidad y a la mayor experiencia con su aplicación.

- Debe señalarse, en términos generales, que la mortalidad, si bien puede disminuir con métodos más sofisticados y técnicamente más complicados, no han influenciado, en gran manera en los resultados; lo más importante es impedir la exanguinación, adecuada reposición de volúmenes, transfusión en el momento oportuno, diagnóstico certero y procedimientos más asequibles.

\section{Tratamiento Farmacológico}

Se emplea los inhibidores de la bomba de protones, en razón de que el aumento del pH promueve la agregación de plaquetas y que el $\mathrm{pH}$ ácido retraza la coagulación dando lugar a la disolución del coágulo, como lo demostró Patchett. El omeprazol en dosis de 80mg por vía intravenosa después del tratamiento endoscópico, seguido de la infusión de 8mg/hora durante 72 horas y de 40mg por vía oral cada 12 horas durante 5 días, reduce en grado significativo la tasa de recurrencia, en pacientes intervenidos quirúrgicamente o en aquellos con vaso sanguíneo visible 


\section{Diagnóstico de las patologías de tubo digestivo y anexos para proceder al manejo clínico y quirúrgico en el tratamiento adecuado}

Vol. 3, núm. 4., (2019)

Karla Valeria Arcentales Vera; Melissa Mercedes Mendieta Torres; César Enrique Vera Sauhing; Diego Israel Ojeda Maldonado

no sangrante o con coágulo adherido, en el caso en que no hayan recibido terapia endoscópica. $\mathrm{Si}$ bien en este último procedimiento, en algunos estudios no ha demostrado beneficios sustanciales, en la práctica debe ser utilizado. (Salón, 2017).

\section{Tratamiento Quirúrgico}

Está reservado, sobre todo para pacientes con hemorragia recurrente, en los que fracasó el tratamiento endoscópico estándar. Tener en cuenta, que la mortalidad operatoria es muy alta, del 5 al 10\%, debido a que los pacientes sometidos a cirugía son generalmente de alto riesgo (edad, cuantía de la hemorragia, cantidad de transfusiones empleadas, enfermedades comórbidas, etc.). Cuando la cirugía está indicada, no debe recurrirse a la observación armada, porque esta pueda ser más bien una actitud por falta de decisión rápida y oportuna tomada por el equipo médico quirúrgico. Es peligroso la exsanguinación y aun el exceso de sangre transfundida. (Salón, 2017).

Fracaso de tratamiento inicial e indicaciones Quirúrgicas

En caso de recidiva, está indicada una segunda gastroscopia y tratamiento endoscópico repetido. Un segundo tratamiento endoscópico es igual de eficaz que la cirugía y tiene menor mortalidad y, en consecuencia, reduce la necesidad de cirugía y el número de complicaciones. Si fracasa o no es posible un segundo tratamiento endoscópico, la angiografía con embolización selectiva es igualmente eficaz y presenta menos efectos adversos que la cirugía; está especialmente indicada en pacientes de alto riesgo quirúrgico. Si no es posible o tampoco se controla la hemorragia, la alternativa final es la cirugía. (Salón, 2017). 


\section{Diagnóstico de las patologías de tubo digestivo y anexos para proceder al manejo clínico y quirúrgico en el tratamiento adecuado}

Vol. 3, núm. 4., (2019)

Karla Valeria Arcentales Vera; Melissa Mercedes Mendieta Torres; César Enrique Vera Sauhing; Diego Israel Ojeda Maldonado

Fármacos y manejo pos-tratamiento Las 3 medidas fundamentales para evitar la recidiva tras la HDA por úlcera péptica son el diagnóstico y tratamiento de la infección por $\mathrm{H}$. pylori y la adecuada gastro protección en pacientes que requieren tomar AINE y recomendaciones sobre anticoagulantes. (Salón, 2017).

Infección por H. pylori: la curación de la infección reduce de manera muy marcada las tasas de recidiva hemorrágica. Por ello, debe investigarse y tratarse la infección en todos los pacientes con SDA por úlcera péptica, duodenitis o gastritis erosiva. Si está presente, se debe tratar y confirmar su erradicación. (Salón, 2017).

Los test para H. pylori (ureasa, test del aliento o biopsia) realizados durante el episodio agudo presentan a menudo resultados falsamente negativos. Esto es probablemente atribuible al tratamiento con IBP administrado al ingreso y quizás a la presencia de sangre en el estómago. Por lo tanto, todo test negativo para H. pylori, realizado en fase aguda de la hemorragia, debe ser confirmado mediante un segundo test diferido realizado en condiciones óptimas Dada la alta prevalencia. (Salón, 2017).

AINE: Los pacientes con historia previa de hemorragia digestiva alta que requieran un AINE deben tratarse con un inhibidor selectivo de la COX-2 (p.ej. celecoxib) asociado a un inhibidor de la bomba de protones. (Salón, 2017)

Anticoagulantes: Después de la gastroscopia, la decisión de reiniciar el tratamiento anticoagulante se debe individualizar. En general, se puede considerar reiniciar la anticoagulación cuando el riesgo de eventos tromboembólicos supera el riesgo de desangrado. Esto sucede 


\section{Diagnóstico de las patologías de tubo digestivo y anexos para proceder al manejo clínico y quirúrgico en el tratamiento adecuado}

Vol. 3, núm. 4., (2019)

Karla Valeria Arcentales Vera; Melissa Mercedes Mendieta Torres; César Enrique Vera

Sauhing; Diego Israel Ojeda Maldonado

habitualmente a las pocas horas tras el tratamiento endoscópico de la lesión sangrante. Durante el ingreso se recomienda iniciar heparina de bajo peso molecular y en casos excepcionales de muy alto riesgo de eventos tromboembólicos (por ejemplo: pacientes portadores de prótesis mitrales metálicas) utilizar heparina sódica. (Salón, 2017).

Se prefiere la heparina a los anticoagulantes porque el efecto anticoagulante de la heparina sódica revierte muy rápidamente al suspender la administración endovenosa. Además, en caso necesario, el efecto anticoagulante puede revertirse con sulfato de protamina. La heparina y bajo peso molecular tiene una vida media algo más larga (alrededor de 12 horas). (Salón, 2017).

Pasos a seguir en el diagnóstico de Patologías de Tubo Digestivo

1. Escuche atentamente las quejas del paciente

- Permita que el paciente se exprese con sus propias palabras.

- Describa la enfermedad actual e inicio de los signos y síntomas.

- Escriba lo más importante en el expediente del paciente.

2. Examen Físico

- Haga su examen físico completo.

- Evalúe al paciente de forma integral.

- Realice evaluación por sistema. 


\section{Diagnóstico de las patologías de tubo digestivo y anexos para proceder al manejo clínico y quirúrgico en el tratamiento adecuado}

Vol. 3, núm. 4., (2019)

Karla Valeria Arcentales Vera; Melissa Mercedes Mendieta Torres; César Enrique Vera Sauhing; Diego Israel Ojeda Maldonado

- Escriba los hallazgos más relevantes en el expediente.

3. Escoja el Signo o Síntoma Relevante Principio o regla general: Si el paciente tiene varios síntomas y signos; escoja el más importante; según el siguiente criterio:

- Escoja un signo (manifestación objetiva de la enfermedad; Ej.: membranas mucosas pálidas) en vez de un síntoma (manifestación subjetiva de la enfermedad: Ej.: fatiga).

- Escoja un signo o síntoma específico que aparece en un restringido número de enfermedades (ej.: dificultad para respirar) en vez de un signo o síntoma general que aparece en muchas enfermedades. (ej.: fiebre)

- Escoja el signo o síntoma reciente más relevante, sin descartar aquellos que están presentes desde hace tiempo. Siguiendo estas instrucciones se puede determinar un problema del signo o síntoma relevante. Anote este síntoma dominante en el expediente del paciente.

4. Determine el Diagnóstico Luego de haber determinado el signo o síntoma dominante, establezca el diagnóstico.

5. Decida el Tratamiento Cuando haya determinado el diagnóstico según el listado, siguiendo las flechas podrá decidir el tratamiento. Hay varias decisiones posibles: 


\section{Diagnóstico de las patologías de tubo digestivo y anexos para proceder al manejo clínico y quirúrgico en el tratamiento adecuado}

Vol. 3, núm. 4., (2019)

Karla Valeria Arcentales Vera; Melissa Mercedes Mendieta Torres; César Enrique Vera

Sauhing; Diego Israel Ojeda Maldonado

- Ciertos pacientes tienen enfermedades muy severas para ser tratados en un centro de salud del Primer Nivel. Este paciente debe ser referido a un hospital.

- Algunas veces se puede empezar el tratamiento o administrar alguna ayuda de emergencia antes de que el paciente sea referido al hospital.

- Para ciertas enfermedades el tratamiento es bastante simple.

- En ocasiones el tratamiento es más complejo.

6. Prescriba un tratamiento y aconseje al paciente

- Prescriba el tratamiento según lo indicado en el diagnóstico o en el programa de tratamientos.

- Explique al paciente cuál es su enfermedad y su tratamiento y si es necesario porque el/ella debe ser referido o debe volver para seguimiento. Además, aconseje sobre nutrición, higiene y cuidado en el hogar.

- Anote el tratamiento y consejos en el expediente del paciente. Notifique a la instancia correspondiente la enfermedad o evento. 


\section{Diagnóstico de las patologías de tubo digestivo y anexos para proceder al manejo clínico y quirúrgico en el tratamiento adecuado}

Vol. 3, núm. 4., (2019)

Karla Valeria Arcentales Vera; Melissa Mercedes Mendieta Torres; César Enrique Vera Sauhing; Diego Israel Ojeda Maldonado

\section{Conclusiones.}

Las enfermedades digestivas son trastornos del aparato digestivo, que algunas veces se denomina tracto gastrointestinal (GI). En la digestión, el alimento y las bebidas se descomponen en pequeñas partes (llamadas nutrientes) que el cuerpo puede absorber y usar como energía y pilares fundamentales para las células. El aparato digestivo está compuesto por el esófago (tubo de alimento), el estómago, los intestinos grueso y delgado, el hígado, el páncreas y la vesícula biliar. Una enfermedad digestiva es cualquier problema de salud que ocurre en el aparato digestivo. Las afecciones pueden ir de leves a serias. Algunos problemas comunes abarcan cáncer, síndrome del intestino irritable e intolerancia a la lactosa.

Los exámenes para los problemas digestivos pueden abarcar colonoscopia, endoscopia de vías digestivas altas, endoscopia con cápsula, colangio pancreatografía retrógrada endoscópica (CPRE) y ecografía endoscópica. Muchos procedimientos quirúrgicos se llevan a cabo en el aparato digestivo. Estos abarcan intervenciones realizadas usando endoscopia, laparoscopia y cirugía abierta. Se pueden realizar trasplantes de órganos en el hígado, el páncreas y el intestino delgado.

Muchos proveedores de atención médica pueden ayudar a diagnosticar y tratar los problemas digestivos. Un gastroenterólogo es un médico especialista que ha recibido formación adicional en el diagnóstico y tratamiento de los trastornos digestivos; esto, considerando que el sistema digestivo juega un papel fundamental en el mantenimiento general de la salud y que la mucosa digestiva es la puerta de entrada de una gran cantidad de antígenos, por ello es importante mantener la integridad y funcionalidad de la misma para que funcione de una manera correcta. 


\section{Diagnóstico de las patologías de tubo digestivo y anexos para proceder al manejo clínico y quirúrgico en el tratamiento adecuado}

Vol. 3, núm. 4., (2019)

Karla Valeria Arcentales Vera; Melissa Mercedes Mendieta Torres; César Enrique Vera Sauhing; Diego Israel Ojeda Maldonado

El tratamiento inicial a seguir en paciente con afecciones del tubo digestivo en inicio se basa enprimordial realizar una historia clínica del paciente ingresado con HD y evaluar su estado hemodinámico (presión arterial, pulso, diuresis), así como conocer la existencia de enfermedades asociadas graves y, muy especialmente, de enfermedad hepática (ictericia, ascitis, encefalopatía). También, hay que preguntar sobre las características del sangrado: hematemesis y/o melenas. Se debe inquirir sobre el antecedente de ingesta previa de fármacos, especialmente AINE, anticoagulantes e ingesta de alcohol.

Posterior a ello, y en base a lo observado se procede a realizar un tratamiento endoscópico, el cual suele considerarse el método más eficaz para el control de la hemorragia ulcerosa aguda y según el aspecto de ella, la prevención de una hemorragia recurrente conforme lo demostró desde hace más de una década Cook en 1992. Reduce la mortalidad, sobre todo en pacientes de alto riesgo como lo ha señalado, la American Society for Gastrointestinal Endoscopy. A pesar de que en manos inexpertas las complicaciones, como agravar la hemorragia o provocar perforaciones puede llegar hasta el 20\%, en servicios especializados estas complicaciones son raras y se sitúan, en o en menos del 1\%. Entre estos procedimientos debe consignarse en orden de posibilidades.

\section{Bibliografía.}

Arias, F. (2010). Paradigmas de la Investigación Científica. España: Luces.

Barret, P. (2015). Métodos de diagnóstico en patologías de Tubo Digestivo. Ciudad de México: McGraw Hill-Interamericana.

Carret, Y. (2015). Manajo Clí́nico de enfermedades de Tubo Digestivo. Revista Médica de la Universidad de la Salud en la ciudad de México(16), 12-23. 


\section{Diagnóstico de las patologías de tubo digestivo y anexos para proceder al manejo clínico y quirúrgico en el tratamiento adecuado}

Vol. 3, núm. 4., (2019)

Karla Valeria Arcentales Vera; Melissa Mercedes Mendieta Torres; César Enrique Vera

Sauhing; Diego Israel Ojeda Maldonado

Comte, B. (2016). Patologías de Tubo Digestivo. Review of the Epidemiology. Medic Informative, $15-18$.

Rentnz, A. (2017). Manejo Clínico y Quirúrgico de Patologías de Tubo Digestivo. Revista Factor Salud de la Universidad Autónoma de México en México, 14-18.

Salón, H. (2017). Manajo diagnóstico de patologías de Tubo Digestivo. Revista de Salud de enfermedades Gástricas. de la Habana Cuba, 15-18.

Ventura, M. (2016). Patologías del Tubo Digestivo, Método Diagnóstico. Revista de Salud Panamericana, 33-35.

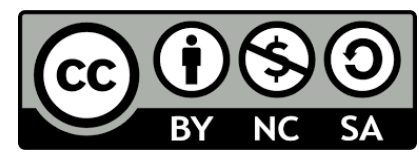

RECONOCIMIENTO-NOCOMERCIAL-COMPARTIRIGUAL

CC BY-NC-SA

ESTA LICENCIA PERMITE A OTROS ENTREMEZCLAR, AJUSTAR Y CONSTRUIR A PARTIR DE SU OBRA CON FINES NO COMERCIALES, SIEMPRE Y CUANDO LE RECONOZCAN LA AUTORÍA Y SUS NUEVAS CREACIONES ESTÉN BAJO UNA LICENCIA CON LOS MISMOS TÉRMINOS. 